\section{Beauty of original experiments}

Landmark Experiments in Twentieth Century Physics. By George L. Trigg. Pp. $x+303$. (Crane Russak: New York; Edward Arnold: London, February 1976.) $£ 11.50$.

IT is a sad fact, but unfortunately a true one, that most physicists' knowledge of the fundamental papers of their subject can only be described as fragmentary, even if one is feeling charitable. How many of us have in fact read the paper by Michelson and Morley, or the verification using $\alpha$ particle scattering of the Rutherford nuclear atom by Geiger and Marsden, or nearer at hand the papers on the de Broglie wavelength of the electron by Davisson and Germer or G. P. Thomson.

We all know of the existence of these experiments and what they prove but our knowledge is second-hand, acquired from the writings of others. It is perhaps not as bad as reading Shakespeare through the eyes of another person, since aesthetic appreciation doesn't enter. If as sometimes happens for a colloquium or lecture course however, we have to examine a bit of fundamental experimentation in detail it is surprising how much rationalisation and perhaps even falsification enters the conventional picture.

This is not deliberate misrepresentation, but it is just that the removal of an awkward corner or fact here and there facilitates understanding and is thus tacitly accepted. For example, one often finds the Michelson-Morley experiment discussed in terms of the time of arrival of pulses of light going down the separate paths. No doubt if the experiment were done this way the result would still be as given and the consequences would be identical; but it is a pity to depart from complete accuracy.

The whole situation stems from the expansion in physics over the past few decades, the need to keep university courses to a sensible length, and the reluctance of physicists to split their subject into more manageable parts. Tiresome examiners at the end of the three undergraduate years demand that we show some powers as practising physicists able to marshal our equations and solve our problems. They are not so concerned as a rule with the way the subject has developed to its present state. Schrödinger's equation, or operators in quantum mechanics, or matrix algebra take precedence over Davisson and Germer, and de Broglie. In this of course they are right, but something important is being lost.
This is true also of books, in which as a particular subject matures so the formal structure grows and the experimental details recede. The present book, however, is of a different kind and attempts to redress the balance. The author takes a list of important twentieth-century experiments, first puts them briefly in their context, and then goes on to describe what was really done in great detail. To do this he uses long sections taken from the original papers which he joins and makes coherent by a minimum of connecting explanation. He thus maintains the authentic flavour of the subject at the time of the experiment; and much of the work is described in the original author's phraseology.

The list of experiments discussed is reasonably well chosen. It begins with the discovery of the wave nature of X-rays using diffraction from crystals, of isotopes, of the concept of atomic number and of superconductivity. During this early period of the century many other possible subjects could of course be chosen but the ones used are not at all unreasonable. It may be that if the book is successful the author might return to this early era and write a second volume on some of the experiments he has omitted.

After this early period, and in fact, coming within the reviewer's memory, the list becomes more restricted and progressively less open to variation. From the early 1930 s to the present day the complete list of topics covers

\section{Nucleon-nucleon forces}

The Nucleon-Nucleon Interaction. By G. E. Brown and A. D. Jackson. Pp. viii +242. (North-Holland: Amsterdam and Oxford; American Elsevier: New York, 1976.) n.p.

WHEN students start research on a particular topic in theoretical physics their first problem is to learn all the essentials of previous work in a finite time. This nearly impossible task is helped by providing them with courses of lectures in which early developments are outlined and more recent work is spelled out in sufficient detail for the student to proceed immediately to the frontiers of the subject equipped to make further progress. G. E. Brown and A. D. Jackson have given such courses in Copenhagen which form the basis of their book. The first four chapters give a simple outline of the wellestablished theory of nucleon interactions at low energies. In chapters 5 and 6 the reader is introduced to techniques in scattering theory, the the fluid properties of liquid helium below the $\lambda$ point, the use of molecular beams for the determination of magnetic moments, the Lamb-Retherford effect, the anomalous $g$ factor for the electron, the transistor, the non-conservation of parity for weak interactions, the Mössbauer effect, the experimental verification of the existence of the neutrino, the maser and laser, tunneling and superconductivity, the $\Omega^{-}$meson and the microwave demonstration of the $3 \mathrm{~K}$ intergalactic radiation temperature.

In spite of its intrinsic interest the book is not easy to read. The subjects cover quite a good cross section of contemporary physics and the use of extracts from the original papers sets a level of difficulty which cannot be entirely overcome by the explanatory connecting parts. This, however, cannot be helped. A student of scientific history or of scientific logic or of experimental technique or anyone just interested in how things really happened could not do better than begin with this book. The reader will get in a few hours an insight which has obviously taken the author a long time and much labour to achieve, and will be left with a feeling that at least some experiments in physics have a beauty which is more akin to art than science.

R. Latham

Dr Latham is a reader in the Department of Physics at Imperial College, University of London, UK.

use of relativistic equations and form factors; a description is also included of a more specialised technique, the eikonalisation of soft vector bosons, which is used by the Copenhagen group to provide convergence.

The final four chapters (half the book) are devoted to a detailed and explicit account of the steps required to derive realistic internucleon potentials and their relativistic corrections from one- and two-boson exchanges. The intimate interweaving of the various pion-nucleon and nucleonnucleon amplitudes is explicitly demonstrated and a clear picture emerges of the physical principles underlying the calculations. This book will be essential reading and remain a basic reference for all those working on nucleon-nucleon forces, as well as being an introduction to the subject for the uninitiated.

Leonardo Castillejo

Leonardo Castillejo is Professor of Physics and head of the theoretical elementary particle physics group in the Department of Physics and Astronomy at University College, London, UK. 